\title{
A mixed-methods assessment of understanding (AoU) tool for AIDS vaccine trials in sub-Saharan Africa: results from a pilot study
}

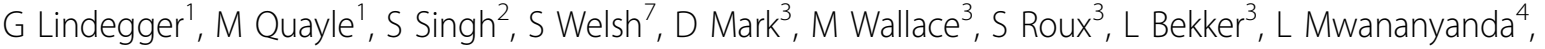 \\ W Kilembe ${ }^{4}$, E Chomba $^{5}$, S Allen ${ }^{6}$, F Priddy $^{7^{*}}$, P Fast $^{7}$
}

From AIDS Vaccine 2012

Boston, MA, USA. 9-12 September 2012

\section{Background}

Assessments of understanding (AoUs) in clinical trials are often composed of true/false multiple choice questions, however, these tools can be difficult for volunteers with limited education or without prior testing experience.

\section{Methods}

35 adults were recruited at two research centers in Southern Africa. A within-subjects, repeated measures design was used, whereby each volunteer served as his/ her own control. An AoU tool with closed- and openended questions was administered within a hypothetical AIDS vaccine trial setting. Performance on closed-and open-ended questions was compared using correlations and repeated-measure t-tests, limited to 4 complex concepts: false sense of security, risk of false positive test, need for contraception, and potentially enhanced susceptibility.

\section{Results}

Mean scores of understanding for each concept assessed by closed-ended questions ranged from 0.73 (need for contraception) to 0.84 (risk of false positive test); and by open-ended questions from 0.4 (risk of false positive test) - 0.6 (need for contraception). Scores for the open-ended measure were all lower than the equivalent closed-ended measure. Correlations between the closedand open-ended measures were generally low, achieving significance for false sense of security $(\mathrm{r}=0.377)$, potentially enhanced susceptibility $(r=0.393)$, and total score across concepts $(\mathrm{r}=0.617)$. Volunteers' understanding as assessed by the closed- and open-ended methods differed significantly: false sense of security $=-3.862$; risk of false positive test $=-7.210$; need for contraception $=$ -2.303 ; and potentially enhanced susceptibility $=-8.007$. The correlation with years of education was consistently and significantly higher for the open-ended measure than the true/false questionnaire with the exception of need for contraception.

\section{Conclusion}

The results suggest the qualitative measure better assesses understanding than the quantitative measure. The scores from the two assessment methods have limited interchangeability. The standard closed-ended questions appear to provide an inflated measure of volunteers' understanding. An assessment tool with closed- and open-ended questions is better suited to determine genuine understanding.

Author details

${ }^{1}$ School of Psychology, University of Kwa-Zulu Natal, Pietermaritzburg, South Africa. ${ }^{2}$ GHAR Consulting Inc, New York, NY, USA. ${ }^{3}$ Desmond Tutu HIV Foundation, University of Cape Town, Cape Town, South Africa. ${ }^{4}$ ZambiaEmory HIV Research Project, Lusaka, Zambia. ${ }^{5}$ Zambia-Emory HIV Reseach Project, Lusaka, Zambia. ${ }^{6}$ Emory University, Atlanta, GA, USA. International AIDS Vaccine Initiative, New York, NY, USA.

Published: 13 September 2012

\section{doi:10.1186/1742-4690-9-S2-P135}

Cite this article as: Lindegger et al: A mixed-methods assessment of understanding (AoU) tool for AIDS vaccine trials in sub-Saharan Africa: results from a pilot study. Retrovirology 2012 9(Suppl 2):P135.

${ }^{7}$ International AIDS Vaccine Initiative, New York, NY, USA

Full list of author information is available at the end of the article

(c) 2012 Lindegger et al; licensee BioMed Central Ltd. This is an Open Access article distributed under the terms of the Creative 